\title{
Rapid progression of skin sclerosis precipitated by a pheochromocytoma in a patient with systemic sclerosis
}

Singapore Med J 2019; 60(8): 436-438 https://doi.org/10.11622/smedj.2019091

Dear Sir,

Systemic sclerosis (SSc) is an immune-mediated disease characterised by endothelial vasculopathy and diffuse fibrosis of internal organs. The extent of skin sclerosis and the accompanying pattern of internal organ involvement are closely related to disease activity. Various nationwide cohort studies have shown that SSC is associated with an elevated incidence of malignancy. ${ }^{(1)}$ Although the role of immunosuppressive therapies and the effects of autoantibodies associated with malignancies are not clearly defined, patients with a later onset of SSc and diffuse cutaneous subtype are at higher risk for malignancy. ${ }^{(2)}$

Pheochromocytoma is a rare tumour associated with high mortality if not diagnosed early. As adrenergic symptoms such as hypertension, headache, palpitations and diaphoresis may be absent, the diagnosis of pheochromocytoma can be missed in cases of atypical presentation. ${ }^{(3,4)}$ There have been very few cases in which a pheochromocytoma mimics SSc or occurs in an SSc patient. ${ }^{(5)}$

In our case, a 57-year-old man presented with hand pain and facial skin thickening that rapidly progressed to the neck and both upper arms over a period of three months. Physical examinations indicate that the patient had Raynaud's phenomenon (RP), which had appeared 12 months before, with sclerodactyly (Fig. 1a). The initial modified Rodnan skin score (mRSS) was 15 points. Nailfold capillaroscopy showed moderate avascularity and dilated capillaries (Fig. 1b \& c). Anti-Scl-70 antibody and antinuclear antibody $(1: 1,280)$ tests were positive. High-resolution computed tomography $(\mathrm{CT})$ was performed to evaluate pulmonary involvement of SSc. However, an incidental large left suprarenal mass was detected and subsequently confirmed on abdominal and pelvic CT (Fig. 2a). PET-CT (positron emission tomography-computed tomography) showed a 5.4-cm hypermetabolic mass in the left adrenal gland (Fig. 2b). The diffusing capacity per litre of lung volume was $77 \%$, forced vital capacity (FVC) was $97 \%$, and forced expiratory volume in one second/FVC was $79 \%$. Other results obtained are shown in Table I. All values were within normal limits.

The patient underwent a laparoscopic adrenalectomy because his elevated normetanephrine levels could have resulted from a pheochromocytoma. A remnant of the adrenal cortex was seen in cross-sections of the adrenal gland, with a 5.5-cm mass showing a solid grey/white surface with haemorrhage and degenerative changes. Microscopy showed polygonal tumour cells
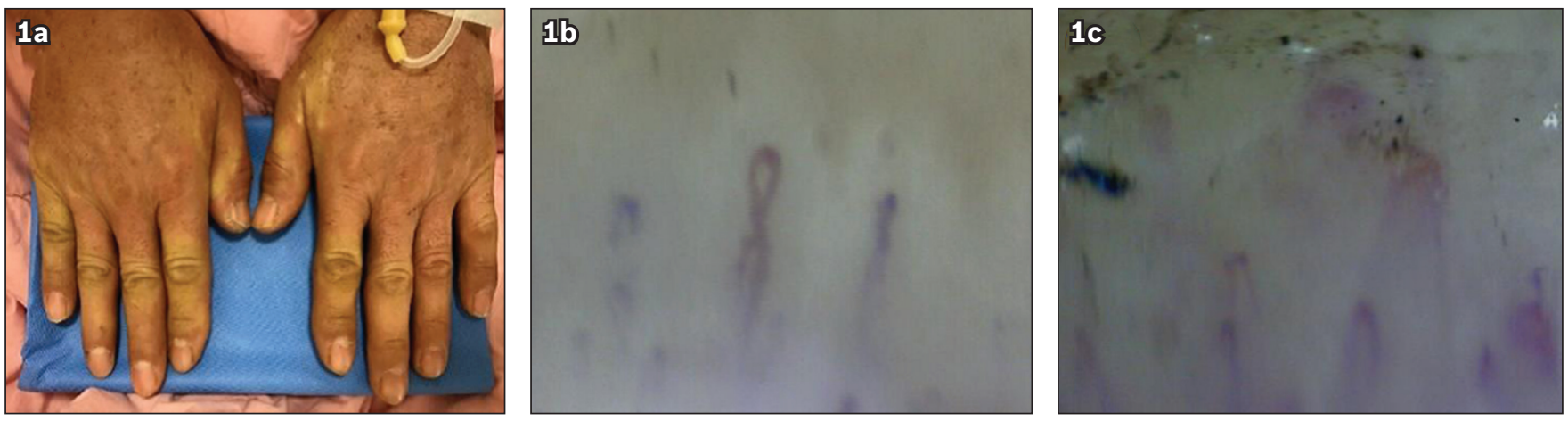

Fig. 1 (a) Photograph shows diffuse oedema, depigmentation and sclerodactyly on both hands. (b \& c) Nailfold capillaroscopy images show microhaemorrhage and a minimal avascular area.
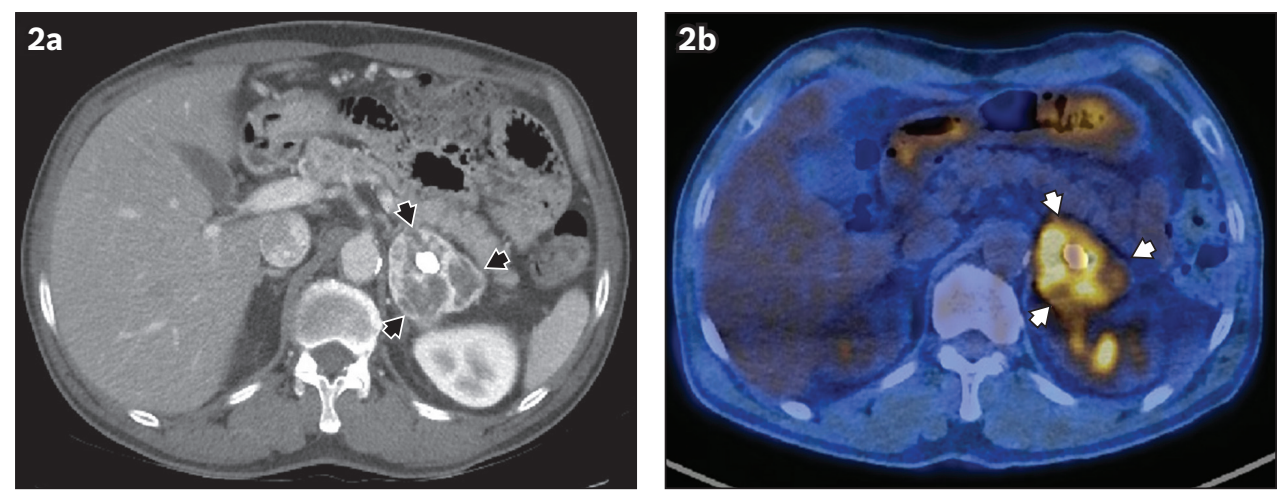

Fig. 2 (a) CT image shows a $5.4 \mathrm{~cm} \times 4.2 \mathrm{~cm}$ enhanced mass with central dense calcification in the left adrenal gland (black arrowheads). (b) PET-CT image of the same area shows a 5.4-cm hypermetabolic mass with calcification in the left adrenal gland (white arrowheads). 
Table I. Test results of the patient.

\begin{tabular}{|lll|}
\hline Parameter & Value & Normal range \\
\hline 24-hour urine vanillyl mandelate $(\mathrm{mg} /$ day $)$ & 3.9 & $0-8.0$ \\
\hline 24-hour urine metanephrine $(\mu \mathrm{g} / \mathrm{day})$ & 55 & $52-341$ \\
\hline 24-hour urine epinephrine $(\mu \mathrm{g} / \mathrm{day})$ & 5.9 & $0-20.0$ \\
\hline 24-hour urine norepinephrine $(\mu \mathrm{g} /$ day $)$ & 77.1 & $15.0-80.0$ \\
\hline Serum epinephrine $(\mathrm{pg} / \mathrm{mL})$ & 17.37 & $0-120.00$ \\
\hline Serum norepinephrine $(\mathrm{pg} / \mathrm{mL})$ & 107.23 & $0-410.00$ \\
\hline Serum metanephrine $(\mathrm{nmol} / \mathrm{L})$ & 0.07 & $0-0.33$ \\
\hline Normetanephrine $(\mathrm{nmol} / \mathrm{L})$ & 2.38 & $0-0.61$ \\
\hline
\end{tabular}
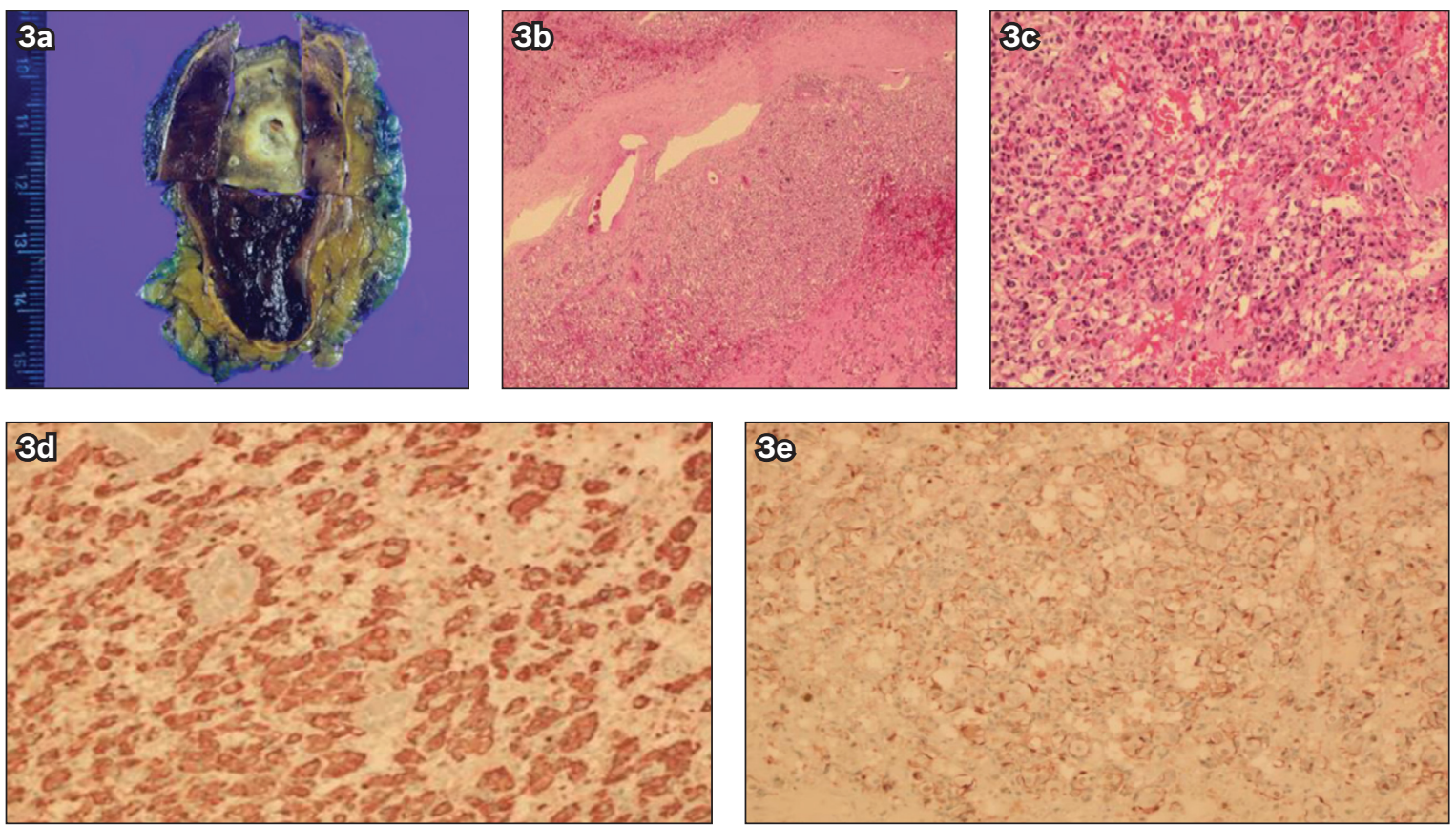

Fig. 3 (a) Cross-sectional photograph shows a remnant of the adrenal cortex with a tumour mass that has a solid grey/white surface with haemorrhage and degenerative changes. Photomicrographs show (b) a well-demarcated capsule and tumour cells with a Zellballen pattern (Haematoxylin \& eosin $[\mathrm{H \& E}], \times 40)$ and $(\mathrm{c})$ the syncytial architecture of the tumour cells with abundant granular cytoplasm (H\&E, $\times 200)$. (d \& e) Photomicrographs with immunohistochemical staining show chromogranin-positive tumour cells $(H \& E, \times 200)$ and S100-protein-positive sustentacular cells $(H \& E, \times 200)$.

with abundant granular cytoplasm arranged in a Zellballen pattern. After staining, chromogranin A and S100 protein tests were positive, the Ki-67 score was 1\%, and the Pheochromocytoma of the Adrenal Gland Scaled Score was less than 4 (Fig. 3). The results of immunohistochemical staining were consistent with those of a pheochromocytoma rather than a malignant tumour. After removal of the pheochromocytoma, the patient's symptoms, which had rapidly deteriorated over the course of three months, did not progress. After one year of routine treatment, his mRSS had decreased from 15 to 9, and there was no recurrence of the pheochromocytoma.

Our case demonstrates rapid progression of skin sclerosis precipitated by a pheochromocytoma in a middle-aged male SSc patient. The patient had RP, one of the most important characteristics of SSc, that was aggravated by vasospasm due to the adrenergic effects of the pheochromocytoma. In such patients, RP can be prolonged and result in ischaemic ulceration or infarction. Although pheochromocytoma rarely presents with acute limb ischaemia, significant negative correlations between plasma concentrations of catecholamine and dermal blood flow have been demonstrated in patients with pheochromocytoma. ${ }^{(4,6)}$ The very high levels of catecholamine production may explain the persistent arterial vasospasm and critical peripheral ischaemia. ${ }^{(6,7)}$ Our patient showed rapid progression of known SSc when incidentally diagnosed with pheochromocytoma, and this may have contributed to the progression of his disease.

Yours sincerely,

Jongtaek $\underline{L e e}^{1}$, Yunsuek $\underline{K i m}^{1}$, Yoonmi $\underline{\text { in}}^{2}$, Hyun-Sook $\underline{\mathrm{Kim}}^{1}$

'Department of Internal Medicine, 'Department of Pathology, Soonchunhyang University Seoul Hospital, College of Medicine, Seoul, Republic of Korea. healthyra@schmc.ac.kr 


\section{Acknowledgement}

This study was supported by funding from Soonchunhyang University, Republic of Korea.

\section{References}

1. Olesen AB, Svaerke C, Farkas DK, Sørensen HT. Systemic sclerosis and the risk of cancer: a nationwide population-based cohort study. Br J Dermatol 2010; 163:800-6.

2. Shah AA, Hummers LK, Casciola-Rosen L, et al. Examination of autoantibody status and clinical features associated with cancer risk and cancer-associated scleroderma. Arthritis Rheumatol 2015; 67:1053-61.

3. Bravo EL. Pheochromocytoma: an approach to antihypertensive management. Ann N Y Acad Sci 2002; 970:1-10.

4. Muehrcke DD, Bliss BP. Early presentation of phaeochromocytoma as acute arterial disease. Br J Hosp Med 1988; 39:534-5.

5. Khoo JJ, Pratt EJ. Phaeochromocytoma mimicking scleroderma. Int J Endocrinol 2011; 2011:917453.

6. Balbir-Gurman A, Menahem Nahir A, Rozin A, Markovits D, Braun-Moscovici Y. Toe necrosis and acute myocardial infarction precipitated by a pheochromocytoma in a patient with systemic sclerosis. J Clin Rheumatol 2007; 13:331-3.

7. Mikasa H, Sakuragi T, Higa K, Yasumoto M. Skin blood flow and plasma catecholamine concentrations during removal of a phaeochromocytoma in a child. Br J Anaesth 2004; 92:757-60. 